\title{
Positional Distribution of DHA in Triacyl-sn-glycerols of Artemia Nauplii Enriched with Salmon Roe Polar Lipids-Containing Fish Oil
}

\author{
Rieko Shiozaki and Yasuhiro Ando* \\ Graduate School of Fisheries Sciences, Hokkaido University \\ (3-1-1 Minato-cho, Hakodate 041-8611, JAPAN)
}

Edited by T. Itoh, Kitasato Univ., and accepted November 11, 2004 (received for review September 13, 2004)

\begin{abstract}
Positional distribution of fatty acids in triacyl-sn-glycerols (TAG) has been investigated for Artemia franciscana nauplii used in marine fish larviculture as an important live food. For enhancement of essential highly unsaturated fatty acids, the nauplii were enriched for 4 to $24 \mathrm{~h}$ with two emulsions containing fish roe polar lipids (PL), (i) a mixture of bonito head oil TAG and salmon roe PL concentrate (88:12, wt/wt) and (ii) an unmixed salmon roe PL concentrate. In TAG isolated from all enriched Artemia nauplii, docosahexaenoic acid (DHA) was preferentially esterified in the $s n-3$ position $(0.7-22.9 \mathrm{~mol} \%)$ followed in sequence by the $s n$ $2(0.1-7.5 \mathrm{~mol} \%)$ and $s n-1(0.0-4.6 \mathrm{~mol} \%)$ positions. This distribution pattern was the same as previously observed for enrichment with bonito head oil TAG free from salmon roe PL. Addition of fish roe PL to DHA-rich fish oil enricher does not seem to practically affect the distribution pattern of DHA characteristic of Artemia nauplii.
\end{abstract}

Key words: Artemia, docosahexaenoic acid (DHA), triacyl-sn-glycerol, polar lipid, positional distribution, enrichment

\section{Introduction}

Artemia nauplii are used in aquaculture as an important live food for marine fish larvae. However, they are deficient in essential n-3 highly unsaturated fatty acids (HUFA), icosapentaenoic acid (IPA) and docosahexaenoic acid (DHA) in particular. In order to ensure successful production of fish larvae, the nauplii are enriched with n-3 HUFA-containing materials prior to being fed to fish larvae (1-3). Marine lipids are typical enrichers of n-3 HUFA for Artemia nauplii (e.g., 4-8).

During the enrichment of Artemia nauplii, DHA mainly increase in triacyl-sn-glycerols (TAG) of the nauplii (7-10). Recently, it was reported that TAG of the enriched Artemia nauplii contained DHA in the $s n-3$ position at a concentration much higher than those in the $s n-1$ and $s n-2$ positions $(11,12)$. This distribution pattern was different from that of marine zooplankton that may form natural diet of marine fish (13). However, previous studies were limited to the nauplii enriched with purified fish oil TAG $(11,14)$ or fatty acid ethyl esters (EE) $(11,12,15)$.

In the present study, Artemia nauplii enriched with two emulsions containing fish roe polar lipids (PL), (i) bonito head oil TAG mixed with salmon roe PL concentrate and (ii) unmixed salmon roe PL concentrate. McEvoy et al. (16) reported that the use of herring roe PL along with DHA-rich tuna orbital oil has advantage

\footnotetext{
${ }^{*}$ Correspondence to: Yasuhiro ANDO, Laboratory of Maine Biosciences Chemistry, Division of Marine Biosciences, Graduate School of Fisheries Sciences, Hokkaido University, 3-1-1 Minato-cho, Hakodate 041-8611, JAPAN

E-mail: ando@fish.hokudai.ac.jp
} 
of nutritionally favorable HUFA valance as well as of their nature such as emulsifier and antioxidant. The aim of the present paper was to supply new data on positional distribution of n-3 HUFA in TAG of the enriched Artemia nauplii and to discuss whether the fish roe PL practically affect the distribution pattern of DHA in Artemia nauplii TAG.

\section{Experimental}

\subsection{Fish Roe PL and Fish Oil TAG Used for Enrichment}

Fish roe PL used for the Artemia enrichment were extracted from salmon roe ("sujiko" in Japanese) and concentrated by a solvent partition procedure using hexane, ethanol and water (17). DHA-rich fish oil TAG were isolated from industrial bonito head oil by column chromatography on Silicagel 60 (Merck, Darmstadt, Germany) with hexane/diethyl ether for elution (18). Fatty acid compositions of the PL concentrate and TAG are shown in Table 1 together with major lipid classes in the PL concentrate. Preparation procedure and detailed lipid profile of these lipids were described in our previous papers $(17,18)$.

Artemia nauplii were enriched with the bonito head oil TAG mixed with salmon roe PL concentrate $(88: 12$, $\mathrm{wt} / \mathrm{wt})(\mathrm{TAG}+\mathrm{PL})$ or unmixed salmon roe PL concentrate. The proportions of TAG and PL in the former were due to the experiment of McEvoy et al. (16), where herring roe PL (12 wt \%) were mixed to tuna orbital oil ( $88 \mathrm{wt} \%)$. For the TAG + PL enrichment, the bonito head oil TAG $(0.88 \mathrm{~g})$ and salmon roe PL concentrate $(0.12 \mathrm{~g})$ were homogenized with $40 \mathrm{ml}$ of distilled water by two-blade blender at 20,000 rpm for 1 min, and for the PL enrichment, the salmon roe PL concentrate $(1.0 \mathrm{~g})$ was homogenized in the similar manner. Resulting emulsions were immediately used for Artemia enrichment.

\section{$2 \cdot 2$ Enrichment of Artemia Nauplii}

Enrichment was carried out with 24-h-old nauplii of Artemia franciscana (INVE, Mountain Green, UT) at a density of 100 nauplii $/ \mathrm{mL}$ in a $10-\mathrm{L}$ tank containing 10 L of well-aerated $20 \%$ salinity artificial seawater at 20 ${ }^{\circ} \mathrm{C}$ (11). The TAG + PL or PL emulsions were added to the tank at the lipid concentration of $100 \mathrm{mg} / \mathrm{L}$. Enriched Artemia nauplii samples (approximately $2 \mathrm{~L}$ of the rearing seawater) were collected on a nylon mesh at 4,18 and $24 \mathrm{~h}$, washed in distilled water, and stored at $-30^{\circ} \mathrm{C}$ prior to lipid analysis within $24 \mathrm{~h}$. Initial samples $(0 \mathrm{~h})$ were also collected just before the start of the enrichment. The 24-h enrichment was carried out in triplicate $(\mathrm{n}=3)$, and each sample was subjected to lipid analysis.

\subsection{Lipid Analysis}

Lipid analysis of the Artemia nauplii was carried out by the procedure previously applied to the analysis of Artemia nauplii $(11,12)$ and rotifers (19). Total lipids (TL) were extracted by the method of Bligh and Dyer (20). TAG were separated from other lipids by preparative thin-layer chromatography on Silicagel 60G plates (0.5 mm thickness; Merck). Fatty acid methyl esters were prepared from the TAG by transesterification on sodium methoxide/methanol catalyst, and analyzed by gas-liquid chromatography on an Omegawax 320 capil-

Table 1 Fatty Acid Composition of Salmon Roe Polar Lipids (PL) Concentrate and Bonito Head Oil Triacyl-sn-glycerols (TAG) Used for Enrichment of Artemia Nauplii (Mol\%).

\begin{tabular}{|c|c|c|}
\hline Fatty acid & $\begin{array}{l}\text { Salmon roe } \\
\text { PL conc. }{ }^{\text {a) }}\end{array}$ & $\begin{array}{l}\text { Bonito head } \\
\text { oil } \mathrm{TAG}^{\mathrm{b})}\end{array}$ \\
\hline 14:0 & 1.1 & 3.2 \\
\hline $16: 0$ & 14.2 & 12.3 \\
\hline iso- $17: 0$ & 1.9 & 7.2 \\
\hline $16: 2 n-4+n-3$ & 0.2 & 1.9 \\
\hline $16: 3 n-4$ & - & 1.0 \\
\hline 18:0 & 12.1 & 1.8 \\
\hline $18: 1 n-9$ & 9.6 & 15.8 \\
\hline $18: 1 n-7$ & 3.6 & 2.8 \\
\hline $18: 2 n-6$ & 1.7 & 2.4 \\
\hline $18: 4 n-3$ & 0.1 & 1.1 \\
\hline $20: 1 n-9$ & 1.2 & 1.0 \\
\hline $20: 4 n-6$ & 2.4 & 2.1 \\
\hline $20: 5 n-3$ & 14.2 & 8.1 \\
\hline $22: 5 n-6$ & - & 1.6 \\
\hline $22: 5 n-3$ & 3.6 & 1.5 \\
\hline $22: 6 n-3$ & 26.9 & 29.3 \\
\hline Others $(<1 \%)$ & 5.6 & 6.9 \\
\hline \multicolumn{3}{|c|}{$\begin{array}{l}\text { a) Reported in reference (17). Lipid classes reported were } \\
\text { phosphatidylcholines }(80 \%) \text {, phosphatidylethanolamines } \\
\text { ( } 7 \% \text { ), phosphatidylinositols }(6 \%) \text {, sterols }(3 \%) \text {, free fatty } \\
\text { acids }(3 \%) \text {, and monoacylglycerols }(1 \%) \text {. }\end{array}$} \\
\hline b) Reported in ref & e (18) & \\
\hline
\end{tabular}


lary column (Supelco, Bellefonte, PA) and a flame ionization detector. Column temperature was programmed from 180 to $240^{\circ} \mathrm{C}\left(1^{\circ} \mathrm{C} / \mathrm{min}\right)$.

Positional distribution of fatty acids in the TAG was also determined by stereospecific analysis previously described $(11,12,19)$. The TAG $(5 \mathrm{mg})$ were partially degraded with ethyl magnesium bromide, and then the resulting 1-, 2- and 3-monoacyl-sn-glycerols (MAG) were isolated by chiral-phase high performance liquid chromatography (HPLC) as their bis-3,5-dinitorophenylurethane derivatives. A column of Sumichiral OA-4100 (Sumitomo Chemical, Osaka, Japan) was used for isolation of $s n-1-$ plus $s n-2-\mathrm{MAG}$ and $s n-3-\mathrm{MAG}$ fractions, and then one of Sumichiral OA-4000 for separation of $s n-1-\mathrm{MAG}$ and $s n-2-\mathrm{MAG}$ fractions. Both HPLC separations were done with hexane/dichloromethane/ethanol $(40: 12: 3, \mathrm{v} / \mathrm{v} / \mathrm{v})$ as mobile phase at $-10^{\circ} \mathrm{C}$. Constituent fatty acids of each fraction were directly analyzed by reversed-phase HPLC on a Capcellpak C18 UG120 S-3 column (Shiseido, Tokyo, Japan) with a linear gradient elution system of $70 \%$ acetonitrile/30\% water to $100 \%$ acetonitrile generated over 90 min. Fatty acid compositions of the $s n-1, s n-2$ and $s n-3$ positions were calculated from the results.

\section{Results and Discussion}

\subsection{TAG Content and Fatty Acid Composi- tion of TAG}

Table 2 shows the TL and TAG contents and fatty acid composition of TAG in the Artemia nauplii. The content of TAG was $6.1 \%$ (initial), 7.3-10.4\% (TAG + PL enrichment) and 3.3-6.1\% (PL enrichment) on dry weight base. The TAG content increased in the nauplii

Table 2 Contents of Total Lipids (TL) and Triacyl-sn-glycerols (TAG) and Fatty Acid Composition of TAG in the Enriched Artemia Nauplii.

\begin{tabular}{|c|c|c|c|c|c|c|c|}
\hline & \multirow{2}{*}{$\begin{array}{c}\text { Initial } \\
0 \mathrm{~h} \\
(\mathrm{n}=4)\end{array}$} & \multicolumn{3}{|c|}{ Enriched with TAG+PL } & \multicolumn{3}{|c|}{ Enriched with $\mathrm{PL}^{\mathrm{b}}$ ) } \\
\hline & & $\begin{array}{c}4 \mathrm{~h} \\
(\mathrm{n}=1)\end{array}$ & $\begin{array}{c}18 \mathrm{~h} \\
(\mathrm{n}=1)\end{array}$ & $\begin{array}{l}24 \mathrm{~h} \\
(\mathrm{n}=3)\end{array}$ & $\begin{array}{c}4 \mathrm{~h} \\
(\mathrm{n}=1)\end{array}$ & $\begin{array}{c}18 \mathrm{~h} \\
(\mathrm{n}=1)\end{array}$ & $\begin{array}{l}24 \mathrm{~h} \\
(\mathrm{n}=3)\end{array}$ \\
\hline \multicolumn{8}{|c|}{ Content ( $\%$, dry weight base) } \\
\hline TL & $15.6 \pm 1.3$ & 17.7 & 21.8 & $17.8 \pm 2.1$ & 16.6 & 14.4 & $12.6 \pm 0.6$ \\
\hline TAG & $6.1 \pm 0.9$ & 7.3 & 10.4 & $7.7 \pm 1.0$ & 6.1 & 4.7 & $3.3 \pm 0.2$ \\
\hline \multicolumn{8}{|c|}{ Fatty acid composition of TAG (Mol\%) } \\
\hline 14:0 & $0.7 \pm 0.6$ & 1.3 & 1.0 & $1.0 \pm 0.0$ & 1.3 & 1.3 & $1.3 \pm 0.0$ \\
\hline iso- $15: 0$ & $1.7 \pm 0.1$ & 1.4 & 0.9 & $0.9 \pm 0.0$ & 1.7 & 1.7 & $1.7 \pm 0.1$ \\
\hline $16: 0$ & $12.6 \pm 0.3$ & 11.8 & 10.5 & $10.6 \pm 0.2$ & 12.4 & 13.0 & $13.0 \pm 0.3$ \\
\hline $16: 1 n-9$ & $1.4 \pm 0.1$ & 1.2 & 1.1 & $1.0 \pm 0.1$ & 1.4 & 1.3 & $1.3 \pm 0.1$ \\
\hline $16: 1 n-7$ & $6.6 \pm 0.1$ & 6.8 & 5.7 & $5.3 \pm 0.2$ & 6.5 & 6.2 & $5.7 \pm 0.3$ \\
\hline $16: 2 n-6$ & $1.1 \pm 0.0$ & 1.0 & 0.8 & $0.7 \pm 0.0$ & 1.1 & 1.2 & $1.2 \pm 0.0$ \\
\hline $17: 1 n-8$ & $1.1 \pm 0.0$ & 1.1 & 1.0 & $1.0 \pm 0.0$ & 1.1 & 1.0 & $1.0 \pm 0.0$ \\
\hline 18:0 & $2.8 \pm 0.1$ & 2.6 & 2.9 & $3.3 \pm 0.2$ & 2.8 & 3.5 & $4.1 \pm 0.4$ \\
\hline $18: 1 n-9$ & $27.0 \pm 0.6$ & 26.4 & 26.3 & $23.9 \pm 0.7$ & 26.2 & 27.3 & $26.5 \pm 0.2$ \\
\hline $18: 1 n-7$ & $5.6 \pm 0.0$ & 5.4 & 5.1 & $5.1 \pm 0.1$ & 5.5 & 5.6 & $5.4 \pm 0.2$ \\
\hline $18: 2 n-6$ & $4.4 \pm 0.1$ & 4.2 & 3.7 & $3.6 \pm 0.1$ & 4.4 & 4.2 & $4.0 \pm 0.1$ \\
\hline $18: 3 n-3$ & $21.1 \pm 0.4$ & 17.6 & 13.3 & $13.0 \pm 0.1$ & 22.2 & 20.4 & $19.4 \pm 1.2$ \\
\hline $18: 4 n-3$ & $2.1 \pm 0.1$ & 1.9 & 1.4 & $1.3 \pm 0.1$ & 2.3 & 1.7 & $1.5 \pm 0.2$ \\
\hline $20: 4 n-6$ & $0.5 \pm 0.0$ & 0.9 & 1.5 & $1.1 \pm 1.0$ & 0.6 & 0.6 & $0.8 \pm 0.1$ \\
\hline $20: 5 n-3$ & $1.8 \pm 0.0$ & 3.3 & 3.4 & $7.1 \pm 0.3$ & 2.0 & 2.2 & $2.7 \pm 0.5$ \\
\hline $22: 6 n-3$ & $0.0 \pm 0.0$ & 4.5 & 11.4 & $11.3 \pm 0.9$ & 0.3 & 0.5 & $1.0 \pm 0.4$ \\
\hline Others $(<1 \%)$ & 9.4 & 8.6 & 10.0 & 9.9 & 8.2 & 8.2 & 9.4 \\
\hline
\end{tabular}

Mean \pm standard deviation $(\mathrm{n}=3$ or 4$)$.

a) Bonito head oil TAG + salmon roe polar lipids concentrate $(88: 12$, wt/wt).

b) Salmon roe polar lipids concentrate $(100 \%)$. 
enriched with TAG + PL, whereas decrease of it was observed for the PL enrichment. In accordance with the changes in TAG content, the TL content also changed.

Concentrations of DHA (22:6n-3) and IPA (20:5n-3) increased in the TAG during both enrichments. With the TAG + PL enrichment, DHA and IPA increased from 0.0 and $1.8 \mathrm{~mol} \%(0 \mathrm{~h})$ to 11.3 and $7.1 \mathrm{~mol} \%(24 \mathrm{~h})$, respectively. In contrast, remarkable decrease of $18: 3 n-$ 3 was observed during this enrichment (from 21.1 to $13.0 \mathrm{~mol} \%$ ). With the PL enrichment, DHA and IPA increased to 1.0 and $2.7 \mathrm{~mol} \%$, respectively, although these levels were not very higher than those of the initial nauplii $(0 \mathrm{~h})$.

\section{$3 \cdot 2$ Positional Distribution of Fatty Acids in TAG}

Table 3 shows the positional distribution of fatty acids in TAG of the Artemia nauplii. Under the analytical conditions used in this study, IPA was not clearly resolved from 16:2n-6. However, content of 16:2n-6 was lower than that of IPA in all of the enriched Artemia nauplii (Table 2). DHA was separated from other major components.

DHA in each position increased in both of the enrichments. In the Artemia nauplii enriched with TAG + PL, concentrations of DHA in the $s n-1, s n-2$ and $s n-3$ positions were 1.7, 3.4 and $8.9 \mathrm{~mol} \%(4 \mathrm{~h}), 4.6,6.9$ and $22.9 \mathrm{~mol} \%(18 \mathrm{~h}$ ), and 4.5, 7.5 and $22.9 \mathrm{~mol} \%(24 \mathrm{~h})$, respectively. In all of the enrichment periods, DHA was esterified in the $s n-3$ position in much preference to the $s n-1$ and $s n-2$ positions. Between the latter two positions, DHA was higher in the $s n-2$ position. With the PL enrichment, this fatty acid was found in the $s n-1, s n$ 2 and $s n-3$ positions at the concentrations of up to 0.3 , 0.6 and $2.3 \mathrm{~mol} \%$ (24 h), respectively. Distribution pattern of DHA in this enrichment was similar to that observed for the TAG + PL enrichment.

IPA with inseparable 16:2n-6 was found in the $s n-1$, $s n-2$ and $s n-3$ positions at concentrations of $4.2,11.2$ and 8.2 mole $\%$ (TAG + PL, $24 \mathrm{~h}$ ), and 1.5, 4.8 and 5.2 mole $\%$ (PL, $24 \mathrm{~h}$ ), respectively. With the TAG + PL enrichment $(24 \mathrm{~h})$, IPA content in TAG was 10 -fold higher than that of 16:2n-6 (Table 2; 7.1 mol\% IPA versus $0.7 \mathrm{~mol} \%$ 16:2n-6). IPA in this Artemia nauplii sample seems to be preferentially esterified in the $s n-2$ position followed by the $s n-3$ and $s n-1$ positions.

The most prominent polyunsaturated fatty acid in the initial nauplii, $18: 3 n-3$, was very high in the $s n-2$ posi- tion (30.8-53.4 mol\%) in both of the initial and enriched Artemia nauplii. Distribution pattern of DHA in the enriched nauplii was not similar to that of $18: 3 n-$ 3.

\section{3·3 Comparison with Previous Data on DHA Distribution}

Recent papers reported the positional distribution of DHA in Artemia nauplii enriched with fish oil TAG or fatty acid EE $(11,12,14,15)$. When TAG of the enriched Artemia nauplii were subjected to regiospecific analysis in order to differentiate the $s n-1,3$ and $s n-2$ positions, DHA was observed in the $s n-1,3$ position higher than in the $s n$-2 position $(14,15)$. When TAG were subjected to stereospecific analysis, this fatty acid appeared to be esterified in the $s n-3$ position in preference to the $s n-1$ and $s n-2$ positions $(11,12)$. The TAG of Artemia nauplii enriched with bonito head oil TAG $(24 \mathrm{~h})$ contained DHA in the $s n-1, s n-2$ and $s n-3$ positions at the concentrations of 7.3, 10.4 and $35.0 \mathrm{~mol} \%$, respectively (11). Similar distribution pattern was found for shorter period enrichment, i.e. 4-h enrichment, 1.5, 2.4 and $7.6 \mathrm{~mol} \%$ and 18-h enrichment, 5.8, 8.9 and $31.4 \mathrm{~mol} \%$ in the $s n$ $1, s n-2$ and $s n-3$ positions, respectively (11).

In the present study, Artemia nauplii were enriched with bonito head oil TAG mixed with salmon roe PL concentrate. The bonito head oil TAG were the same one used in the previous study (11). The enrichment of Artemia nauplii was also carried out under the same conditions except for no use of gelatin as an emulsifier of TAG. DHA in the enriched Artemia nauplii appeared to be preferentially esterified in the $s n-3$ position followed by the $s n-2$ and $s n-1$ positions. This result was similar to the distribution pattern found in the previous enrichment without salmon roe PL. Therefore, the salmon roe PL concentrate added to bonito head oil TAG does not seem to affect the positional distribution pattern of DHA in the enriched Artemia nauplii.

Phospholipids have no fatty acid in the $s n-3$ position. DHA in fish roe phosphatidylcholines, major lipid class in fish roe PL, is generally esterified in the $s n-2$ position of their glycerol backbones (1). However, it is not probable that this distribution pattern hold for TAG of the enriched Artemia nauplii. In the present study, unmixed salmon roe PL concentrate was also used for the enrichment of Artemia nauplii. High preference of DHA in the $s n-3$ position was also found in this enrichment. 


\subsection{DHA Distribution Characteristic of Artemia Nauplii}

Previously, McEvoy et al. (16) enriched Artemia nauplii with tuna orbital oil mixed with herring roe PL (88:12, wt/wt). After 18-h enrichment, DHA and IPA contents found in the enriched nauplii were 19.4 and
$10.8 \mathrm{mg} / \mathrm{g}$ (dry weight base), respectively, corresponding to 14.1 and $7.8 \%$ of total fatty acids. The ratio of DHA/IPA (1.8) was similar to those (approximately 2.0) found in yolk of wild fish eggs and in copepods, natural prey of most marine fish larvae $(1,3,16)$. Based on the results of Table 2, DHA and IPA in the Artemia nauplii enriched with TAG + PL were calculated to be

Table 3 Positional Distribution of Fatty Acids in Triacyl-sn-glycerols (TAG) of the Enriched Artemia Nauplii (Mol\%).

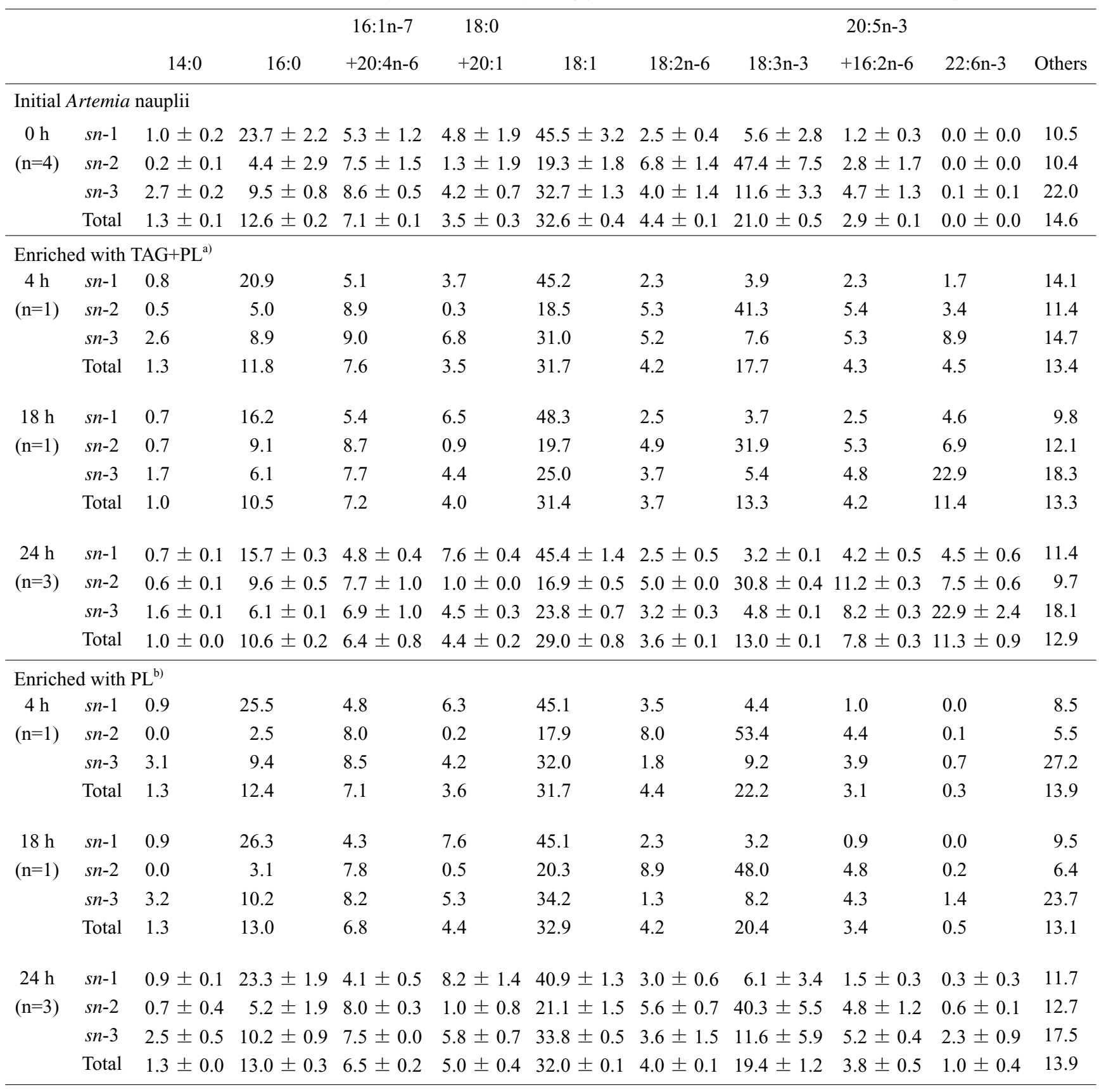

Mean \pm standard deviation ( $\mathrm{n}=3$ or 4 ).

a) Bonito head oil TAG + salmon roe polar lipids concentrate $(88: 12, \mathrm{wt} / \mathrm{wt})$.

b) Salmon roe polar lipids concentrate $(100 \%)$. 


\section{R. Shiozaki and Y. Ando}

11.9 and $3.5 \mathrm{mg} / \mathrm{g}(18 \mathrm{~h})$ and 8.7 and $5.5 \mathrm{mg} / \mathrm{g}(24 \mathrm{~h})$, respectively. These levels were about one half of those reported (16), probably resulting from differences not only in lipid classes analyzed (TL versus TAG) but also in enrichment protocols, e.g., age of the initial nauplii, temperature of the rearing seawater, dose of enricher, and the number of dosing times. However, the DHA/IPA ratio around 2.0 could be achieved in the present study. When calculated from Table 2, the ratio was $3.4(18 \mathrm{~h})$ and $1.6(24 \mathrm{~h})$.

The Artemia nauplii enriched with the herring roe PL-containing tuna orbital oil were recognized to be nutritionally closer to wild prey than those enriched with other diet $(1,3,16)$. The enriched Artemia nauplii led to satisfactory (though not necessary optimal) survival, growth, pigmentation and metamorphosis of turbot larvae (3). On the other hand, including the present study, there have been no data showing that DHA is preferentially esterified in the $s n-2$ position of Artemia TAG. This is opposite to TAG of natural marine fish eggs or marine zooplankton where DHA is highest in the $s n-2$ position (13). Even though fish roe PL rich in DHA in the $s n-2$ position are used as a part of HUFA enricher, Artemia nauplii seem to incorporate DHA into the $s n-3$ position of their TAG. Therefore, positional distribution of DHA in TAG of Artemia nauplii is strongly characterized by high preference in the $s n-3$ position to the other two positions.

\section{References}

1. J.R. SARGENT, D.R. TOCHER and J.G. BELL, The Lipids, in Fish Nutrition, 3rd edn., (J.E. HALVER and R.W. HARDY, ed.), Academic Press, San Diego, pp. 181-257 (2002).

2. P. SORGELOOS, P. DHERT and P. CANDREVA, Use of the Brine Shrimp, Artemia spp., in Marine Fish Larviculture, Aquaculture, Vol. 200, 147-159 (2001).

3. J. SARGEnt, L. MCEVoy, A. ESteVez, G. BelL, M. BELL, J. HENDERSON and D. TOCHER, Lipid Nutrition of Marine Fish Early Development: Current Status and Future Directions, Aquaculture, Vol. 179, 217-229 (1999).

4. J.R. RAINUZZO, K.I. REITAN, L. JØRGENSEN and Y. OLSEN, Lipid Composition in Turbot Larvae Fed Live Feed Culture by Emulsions of Different Lipid Classes, Comp. Biochem. Physiol., Vol. 107A, 699-710 (1994).

5. L.A. MCEVOY, J.C. NAVARRO, J.G. BELL and J.R. SARGENT, Autoxidation of Oil Emulsions during the Artemia Enrichment Process, Aquaculture, Vol. 134, 101-112 (1995).

6. P.C. SOUTHGATE and D.C. LOU, Improving the n-3 HUFA Composition of Artemia Using Microcapsules Containing
Marine Oils, Aquaculture, Vol. 134, 91-99 (1995).

7. P. COUTTEAU and G. MOURENTE, Lipid Classes and their Content of n-3 Highly Unsaturated Fatty Acids (HUFA) in Artemia franciscana after Hatching, HUFA-Enrichment and Subsequent Starvation, Mar. Biol., Vol. 130, 81-91 (1997).

8. K. HAN, I. GEURDEN and P. SORGELOOS, Enrichment Strategies for Artemia Using Emulsions Providing Different Levels of n-3 Highly Unsaturated Fatty Acids, Aquaculture, Vol. 183, 335-347 (2000).

9. J.C. NAVARRO, R.J. HENDERSON, L.A. MCEVOY, M.V. BELL and F. AMAT, Lipid Conversions during Enrichment of Artemia, Aquaculture, Vol. 174, 155-166 (1999).

10. T. TAKEUCHI, M. TOYAMA and T. WATANABE, Comparison of Lipid and n-3 Highly Unsaturated Fatty Acid Incorporations between Artemia Enriched with Various Types of Oils by Direct Methods, Nippon Suisan Gakkaishi, Vol. 58, 277-281 (1992).

11. Y. ANDO, Y. MURAYAMA and H. SAMOTO, Positional Distribution of DHA and EPA in Triacyl-sn-glycerols (TAG) of Artemia franciscana Nauplii Enriched with Fish Oils Ethyl Esters and TAG, Aquaculture, Vol. 233, 321-335 (2004).

12. Y. ANDO and Y. OOMI, Positional Distribution of Highly Unsaturated Fatty Acids in Triacyl-sn-glycerols of Artemia Nauplii Enriched with Docosahexaenoic Acid Ethyl Ester, Lipids, Vol. 36, 733-739 (2001).

13. H. BROCKERHOFF, M. YURKOWSKI, R.J. HOYLE and R.G. ACKMAN, Fatty Acid Distribution of Marine Plankton, J. Fish. Res. Board Can., Vol. 21, 1379-1384 (1964).

14. Y. ANDO and K. NARUKAWA, Regiospecific Distribution of Highly Unsaturated Fatty Acids in Triacylglycerols of Artemia Nauplii Enriched with Marine Oils, J. Oleo Sci., Vol. 51, 615620 (2002).

15. Y. ANDO, Y. OOMI and K. NARUKAWA, Regiospecific Distribution of Fatty Acids in Triacylglycerols of Artemia franciscana Nauplii Enriched with Fatty Acid Ethyl Esters, Comp. Biochem. Physiol., Vol. 133B, 191-199 (2002).

16. L.A. MCEVOY, J.C. NAVARRO, F. HONTORIA, F. AMAT and J.R. SARGENT, Two Novel Artemia Enrichment Diets Containing Polar Lipid, Aquaculture, Vol. 144, 339-352 (1996).

17. Y. ANDO, H. SAMOTO and S. SASAKI, Preparation of Salmon Roe Polar Lipid Concentrate as an HUFA Enricher of Artemia Nauplii, J. Oleo Sci., Vol. 52, 443-447 (2003).

18. Y. ANDO, M. SATAKE and Y. TAKAHASHI, Reinvestigation of Positional Distribution of Fatty Acids in Docosahexaenoic Acid-Rich Fish Oil Triacyl-sn-glycerols, Lipids, Vol. 35, 579582 (2000).

19. Y. ANDO, S. KOBAYASHI, T. SUGIMOTO and N. TAKAMARU, Positional Distribution of $n-3$ Highly Unsaturated Fatty Acids in Triacyl-sn-glycerols (TAG) of Rotifers (Brachionus plicatilis) Enriched with Fish and Seal oils TAG, Aquaculture, Vol. 233, 321-335 (2004).

20. E.G. BLIGH and W.J. DYER, A Rapid Method of Total Lipid Extraction and Purification, Can. J. Biochem. Physiol., Vol. 37, 911-917 (1959). 